\title{
Bridging scale methods for nanomechanics and materials
}

\author{
Wing Kam Liu ${ }^{\text {a,*, Harold S. Park }}{ }^{\text {b }}$, Dong Qian ${ }^{\text {c }}$, Eduard G. Karpov ${ }^{\text {d, }}$ \\ Hiroshi Kadowaki ${ }^{\text {e, Gregory J. Wagner }}{ }^{\mathrm{f}}$ \\ a Department of Mechanical Engineering, The Technological Institute, Northwestern University, 2145 Sheridan Road, Evanston, \\ IL 60208-3111, United States \\ ${ }^{\mathrm{b}}$ Department of Civil and Environmental Engineering, Vanderbilt University, Nashville, TN 37235-1831, United States \\ ${ }^{\mathrm{c}}$ Department of Mechanical, Industrial and Nuclear Engineering, University of Cincinnati, Cincinnati, OH 45221, United States \\ d Department of Mechanical Engineering, Northwestern University, Evanston, IL 60208, United States \\ ${ }^{\mathrm{e}}$ Bridgestone Corporation, Tokyo, Japan \\ ${ }^{\mathrm{f}}$ Sandia National Laboratories, Livermore, CA 94551, United States
}

Received 10 November 2004; received in revised form 13 January 2005; accepted 9 May 2005

\begin{abstract}
Inspired by the pioneering work of Professor T.J.R. Hughes on the variational multi-scale method, this document summarizes recent developments in multiple-scale modeling using a newly developed technique called the bridging scale. The bridging scale consists of a two-scale decomposition in which the coarse scale is simulated using continuum methods, while the fine scale is simulated using atomistic approaches. The bridging scale offers unique advantages in that the coarse and fine scales evolve on separate time scales, while the high frequency waves emitted from the fine scale are eliminated using lattice impedance techniques. Recent advances in extending the bridging scale to quantum mechanical/continuum coupling are briefly described. The method capabilities are demonstrated via quasistatic nanotube bending, dynamic crack propagation and dynamic shear banding.
\end{abstract}

(C) 2005 Elsevier B.V. All rights reserved.

Keywords: Multiple scale simulations; Bridging scale; Finite temperature; Coupling methods; Molecular dynamics; Finite elements; Generalized Langevin equation; Carbon nanotubes; Dynamic fracture; Quantum mechanical/continuum coupling

\footnotetext{
${ }^{*}$ Corresponding author. Tel.: +1 847491 7094; fax: +1 8474913915.

E-mail address: w-liu@northwestern.edu (W.K. Liu).
} 


\section{Introduction}

The rapid advances in nanotechnology, nanomaterials and nanomechanics offer huge potentials in national defense, homeland security, and private industry. An emphasis on nanoscale entities will make our manufacturing technologies and infrastructure more sustainable in terms of reduced energy usage and environmental pollution. Recent advances in the research community on this topic have stimulated ever-broader research activities in science and engineering devoted to their development and their applications.

With the confluence of interest in nanotechnology, availability of experimental tools to synthesize and characterize systems in the nanometer scale, and computational tools widely accessible to model microscale systems by coupled continuum/molecular/quantum mechanics, we are poised to unravel the traditional gap between the atomic and the macroscopic world in mechanics and materials. This in turn opens up new opportunities in education and research. Over the past three decades, we have acquired new tools and techniques to synthesize nanoscale objects and to learn their many incredible properties. Today's high-resolution electron microscopes can routinely see individual atoms. Scanning probe techniques allow us to manipulate atoms one at a time. Advanced materials synthesis provides the technology to tailordesign systems from as small as molecules to structures as large as the fuselage of a plane. We now have the technology to detect single molecules, bacteria or virus particles. We can make protective coatings more wearresistant than diamond and fabricate alloys and composites stronger than ever before.

In most of these applications, nanoscale materials will be used in conjunction with other components that are larger, and have different response times, thus operating at different time and length scales. Single scale methods such as ab initio quantum mechanical methods or molecular dynamics (MD) will have difficulty in analyzing such hybrid structures due to the limitations in terms of the time and length scales that each method is confined to. Because of the availability of accurate interatomic potentials for a range of materials, classical MD simulations have become prominent as a tool for elucidating complex physical phenomena. However, the length and time scales that can be probed using MD are still fairly limited. For the study of nanoscale mechanics and materials, we must model up to a scale of several microns, consisting of billions of atoms, which is too large for MD simulations to-date. Hence, we need to develop multi-scale approaches for this class of problems.

One possible approach that can be applied to many problems is to use MD only in localized regions in which the atomic-scale dynamics are important, and a continuum simulation method, such as the finite element (FE) [1,2] or meshfree methods [3-5] everywhere else. This general approach has been taken by several different groups using methods that have had varying degrees of success. In particular, these methods do not satisfactorily address the issue of disparate time scales in the two regions, and provide a simplified treatment of the interface between the atomistic and continuum regimes. For a more complete review of available multiple scale methods, see Liu et al. [6], or Curtin and Miller [7].

Among Professor T.J.R. Hughes' many important contributions to the field of solid mechanics was his work on the variational multi-scale method. In that pioneering work [8], the displacement field was decomposed into coarse and fine scale components. The fine scale component was used to resolve a local field of interest using an element level enrichment. This work has motivated an alternative multi-scale approach, the bridging scale, which was developed in a seminal work by Wagner and Liu [9] using a similar coarse/fine decomposition of the displacement field. In the bridging scale, the continuum representation exists everywhere in the domain, including those areas in which MD is present. By using a projection operator to decompose the displacement field into orthogonal coarse and fine scales, they were able to derive a coupled set of equations of motion describing the evolution of the MD and FE systems.

These coupled but separate equations of motion have three major benefits. The first is that the FE and MD equations of motion are not required to be integrated using the same timestep. The second benefit is that it allows the elimination of unwanted MD degrees of freedom by accounting for them in the form of an 
impedance force which augments the standard MD equations of motion. Because those unwanted MD degrees of freedom are accounted for in a mathematically consistent manner, high frequency waves which cannot be represented by the continuum mesh are dissipated naturally out of the MD region. Lastly, the bridging scale is valid for finite-temperature dynamic problems.

The purpose of this paper is to introduce the bridging scale method, then demonstrate applications of the method, as well as present new research developments. We will highlight two recent advances; the first concerns the development of a method by Karpov et al. [10] which allows the finite temperature modeling of the atomistic domain in coupled atomistic/continuum simulations. The second major development is the work of Qian and Liu [11,12], which couples a quantum mechanical method, the tight-binding (TB) method, to a continuum representation for the quasistatic analysis of nanomaterials.

The outline of this paper is as follows. We first review the bridging scale, giving special detail to the coupled coarse and fine scale equations of motion, as well as the mathematical elimination of unwanted fine scale degrees of freedom. We then demonstrate the performance of the method on such nonlinear problems as quasistatic nanotube bending, dynamic atomistic crack propagation and dynamic shear banding. We conclude this article by summarizing the current state of the bridging scale, and commenting on directions of future research.

\section{Bridging scale fundamentals}

The bridging scale was recently developed by Wagner and Liu [9] to concurrently couple atomistic and continuum simulations. By concurrent coupling, we imply that both simulations run simultaneously while dynamically exchanging relevant information. Because much of the following has already been derived in detail (see Wagner and Liu [9] and Park and Liu [13]), we refer the interested reader to those works for further details. The fundamental idea is to decompose the total displacement field $\mathbf{u}(\mathbf{x})$ into coarse and fine scales

$$
\mathbf{u}(\mathbf{x})=\overline{\mathbf{u}}(\mathbf{x})+\mathbf{u}^{\prime}(\mathbf{x}) .
$$

This decomposition has been used before in solid mechanics, for example by Hughes et al. [8] in the variational multi-scale method. The coarse scale $\overline{\mathbf{u}}$ is that part of the solution which can be represented by a set of basis functions, i.e. finite element or meshfree shape functions. The fine scale $\mathbf{u}^{\prime}$ is defined as the part of the total solution whose projection onto the coarse scale is zero.

In order to describe the bridging scale, we imagine a body in any dimension which is described by $N_{a}$ atoms. The total displacement of an atom $\alpha$ is written as $\mathbf{u}_{\alpha}$. The coarse scale displacement is a function of the initial positions $\mathbf{X}_{\alpha}$ of the atoms, which implies a Lagrangian description of the domain. It should be noted that the coarse scale would at first glance be thought of as a continuous field, since it can be interpolated between atoms using finite element shape functions. However, because the fine scale is defined only at atomic positions, the total displacement and thus the coarse scale are discrete functions that are defined only at atomic positions. For consistency, Greek indices $(\alpha, \beta, \ldots)$ will define atoms for the remainder of this paper, and uppercase Roman indices $(I, J, \ldots)$ will define coarse scale nodes.

The coarse scale is defined to be

$$
\overline{\mathbf{u}}\left(\mathbf{X}_{\alpha}\right)=\sum_{I} N_{I}^{\alpha} \mathbf{d}_{I}
$$

Here, $N_{I}^{\alpha}=N_{I}\left(\mathbf{X}_{\alpha}\right)$ is the shape function of node $I$ evaluated at point $\mathbf{X}_{\alpha}$, and $\mathbf{d}_{I}$ is the FE nodal displacement associated with node $I$.

The fine scale is defined to be the projection of the coarse scale subtracted from the total solution $\mathbf{u}_{\alpha}$. In other words, the fine scale represents that part of the total solution that the coarse scale cannot represent. 
For our purposes, we shall obtain the fine scale information from displacements q obtained from a molecular dynamics (MD) simulation. We will select this projection operator to minimize the mass-weighted square of the fine scale, which can be written as

$$
J=\sum_{\alpha} m_{\alpha}\left(\mathbf{q}_{\alpha}-\sum_{I} N_{I}^{\alpha} \mathbf{w}_{I}\right)^{2},
$$

where $m_{\alpha}$ is the atomic mass of an atom $\alpha$ and $\mathbf{w}_{I}$ are temporary coarse scale degrees of freedom. It should be emphasized that (3) is only one of many possible ways to define an error metric. The error $J$ is minimized and the resulting fine scale is recombined with the coarse scale to obtain the total displacement as

$$
\mathbf{u}=\mathbf{N d}+\mathbf{q}-\mathbf{P q},
$$

where

$$
\mathbf{P}=\mathbf{N} \mathbf{M}^{-1} \mathbf{N}^{\mathrm{T}} \mathbf{M}_{\mathrm{A}}
$$

defines a projection matrix, $\mathbf{M}_{\mathrm{A}}$ is a diagonal atomic mass matrix and $\mathbf{M}$ is the consistent finite element mass matrix. The final term in (4) is called the bridging scale. It is the part of the solution that must be removed from the total displacement so that a complete separation of scales is achieved, i.e. the coarse and fine scales are orthogonal, or linearly independent of each other.

\subsection{Multi-scale equations of motion}

The next step in the multi-scale process is to derive the coupled MD and FE equations of motion. This is done by first constructing a Lagrangian $\mathscr{L}$, which is defined to be the kinetic energy minus the potential energy

$$
\mathscr{L}(\mathbf{u}, \dot{\mathbf{u}})=\mathscr{K}(\dot{\mathbf{u}})-V(\mathbf{u}) .
$$

Substituting the time derivative of (4) into the Lagrangian (6) gives

$$
\mathscr{L}(\mathbf{d}, \dot{\mathbf{d}}, \mathbf{q}, \dot{\mathbf{q}})=\frac{1}{2} \dot{\mathbf{d}}^{\mathrm{T}} \mathbf{M} \dot{\mathbf{d}}+\frac{1}{2} \dot{\mathbf{q}}^{\mathrm{T}} \mathscr{M} \dot{\mathbf{q}}-U(\mathbf{d}, \mathbf{q}),
$$

where the fine scale mass matrix $\mathscr{M}$ is defined to be $\mathscr{M}=\mathbf{Q}^{\mathrm{T}} \mathbf{M}_{\mathrm{A}}$, with $\mathbf{Q} \equiv \mathbf{I}-\mathbf{P}$. One elegant feature of (7) that results due to the choice of error metric (3) is that the total kinetic energy has been decomposed into the sum of the coarse scale kinetic energy plus the fine scale kinetic energy.

The multi-scale equations of motion are obtained from the Lagrangian by following the relations

$$
\begin{aligned}
& \frac{\mathrm{d}}{\mathrm{d} t}\left(\frac{\partial \mathscr{L}}{\partial \dot{\mathbf{d}}}\right)-\frac{\partial \mathscr{L}}{\partial \mathbf{d}}=0, \\
& \frac{\mathrm{d}}{\mathrm{d} t}\left(\frac{\partial \mathscr{L}}{\partial \dot{\mathbf{q}}}\right)-\frac{\partial \mathscr{L}}{\partial \mathbf{q}}=0 .
\end{aligned}
$$

Substituting the Lagrangian (7) into (8) and (9) gives the coupled multi-scale equations of motion:

$$
\begin{aligned}
& \mathbf{M}_{\mathrm{A}} \ddot{\mathbf{q}}=\mathbf{f}(\mathbf{u}), \\
& \mathbf{M} \ddot{\mathbf{d}}=\mathbf{N}^{\mathrm{T}} \mathbf{f}(\mathbf{u}) .
\end{aligned}
$$

Eqs. (10) and (11) define the coupled multi-scale equations of motion. As can be seen, (10) is simply the MD equation of motion. Therefore, a standard MD solver can be used to obtain the MD displacements q, while the MD forces $\mathbf{f}$ can be found using any relevant potential energy function. Furthermore, we can use standard finite element methods to find the solution to (11). One important note is that the finite element mass 
matrix $\mathbf{M}$ is defined to be a consistent mass matrix. It is also crucial to note that while the MD equation of motion is only solved in the MD region, the FE equation of motion is solved everywhere.

The coupling between the two equations is through the coarse scale internal force $\mathbf{N}^{\mathrm{T}} \mathbf{f}(\mathbf{u})$, which is a direct function of the MD internal force $\mathbf{f}$. In the region in which MD exists, the coarse scale force is calculated by extrapolating the MD force. The MD forces can therefore be thought of as defining the constitutive relation for the finite element internal force. In the region in which MD has been eliminated, the coarse scale force can be calculated in multiple ways. Details are provided in Wagner and Liu [9], Park and Liu [13] and Qian et al. [14].

Because the coarse scale is defined to be the projection of the fine scale upon the coarse scale (FE) basis functions, the FE equation of motion (11) is simply the projection of the MD equation of motion (10) on the FE basis functions. To see that this is so, when the FE mesh is graded down such that a one-to-one correspondence exists between atoms and nodes, the FE equation of motion (11) exactly converges to the MD equation of motion (10). In the coarse scale limit, where the FE nodal spacing is much larger than the atomic spacing, the FE equation of motion represents an approximation to the MD equation of motion by way of the FE shape functions.

We note that the FE equation of motion is redundant for the case in which the MD and FE regions both exist everywhere in the domain, because the FE equation of motion is simply an approximation to the MD equation of motion. We shall remove this redundancy in the next section, when we create coupled MD/FE equations of motion for systems where the MD region is confined to a small portion of the domain.

\subsection{Elimination of unwanted fine scale degrees of freedom}

In order to eliminate the unwanted fine scale degrees of freedom, we decompose (10) into coarse and fine components, and rewrite the fine scale equation in component form as

$$
\ddot{\mathbf{u}}_{l, m, n}^{\prime}(t)=\sum_{l^{\prime}, m^{\prime}, n^{\prime}} \mathbf{M}_{\mathrm{A}}^{-1} \mathbf{K}_{l-l^{\prime}, m-m^{\prime}, n-n^{\prime}} \mathbf{u}_{l^{\prime}, m^{\prime}, n^{\prime}}^{\prime}(t)+\mathbf{M}_{\mathrm{A}}^{-1} \mathbf{f}_{l, m, n}^{\mathrm{ext}}(t)
$$

where $\mathbf{f}_{l, m, n}^{\text {ext }}(t)$ is the external force acting upon unit cell $(l, m, n)$, the constant stiffness matrices $\mathbf{K}$ relate the displacements in cell $\left(-l^{\prime},-m^{\prime},-n^{\prime}\right)$ to the forces in cell $(l, m, n)$ and the size of the summation represents the range of the forces in the $m$ and $n$ coordinate directions. If longer ranged interactions are desired, this approximation can be relaxed simply by increasing the interaction range of the $\mathbf{K}$ matrices, as shown in Park et al. [15].

Using the standard techniques of Laplace and Fourier transforms as described in Wagner et al. [16], Park et al. [17] and Karpov et al. [18], the unwanted fine scale degrees of freedom are eliminated while keeping the effects of the unwanted fine scale degrees of freedom on the remaining lattice. The elimination of the unwanted MD degrees of freedom is made possible due to the repetitive and periodic nature of regular crystal lattices. By utilizing the periodicity of the lattice structure, the final form for the coupled MD and FE equations of motion can be written as

$$
\begin{aligned}
& \mathbf{M}_{\mathrm{A}} \ddot{\mathbf{q}}(t)=\mathbf{f}(t)+\mathbf{f}_{0, m, n}^{\mathrm{imp}}(t)+\mathbf{R}_{0, m, n}^{\mathrm{f}}(t), \\
& \mathbf{M} \mathbf{d}=\mathbf{N}^{\mathrm{T}} \mathbf{f}(\mathbf{u}),
\end{aligned}
$$

where

$$
\mathbf{f}_{0, m, n}^{\mathrm{imp}}(t)=\sum_{m^{\prime}, n^{\prime}} \int_{0}^{t} \boldsymbol{\theta}_{m-m^{\prime}, n-n^{\prime}}(t-\tau)\left(\mathbf{q}_{0, m^{\prime}, n^{\prime}}(\tau)-\overline{\mathbf{u}}_{0, m^{\prime}, n^{\prime}}(\tau)-\mathbf{R}_{0, m^{\prime}, n^{\prime}}^{\mathrm{d}}(\tau)\right) \mathrm{d} \tau .
$$

Eqs. (13)-(15) represent the major results of this section. With these key equations having been derived, several remarks are in order: 
- The first equation (13) defines the standard MD equation of motion, with key modifications. These modifications will be described in detail in the following exposition. It should be noted that the interatomic forces $\mathbf{f}(t)$ can be derived from any anharmonic potential energy function.

- The second term on the right-hand side of $(13), \mathbf{f}_{0, m, n}^{\text {imp }}(t)$, contains the time history kernel $\boldsymbol{\theta}(t-\tau)$, and acts to dissipate fine scale energy from the MD simulation into the surrounding continuum. We call this external force the impedance force, which acts on the reduced MD system due to the eliminated fine scale degrees of freedom. The numerical result is a non-reflecting boundary between the MD and FE regions, as the time history kernel allows short wavelengths that cannot be represented by the surrounding continuum to leave the MD region. It is this term that plays the main role in distinguishing the bridging scale from previous concurrent multi-scale approaches, as the ability of the method to dissipate high frequency information which cannot be captured by the coarse scale can be demonstrated to be clearly and unequivocably tied to the proper implementation of this term $[9,13,17]$. This second term acts only on the MD boundary atoms; away from the MD boundary, this term is zero and the standard MD equations of motion result.

- It is important to note that all assumptions of linearity are completely contained within the time history kernel $\boldsymbol{\theta}(t)$. The assumption of linearity arises due to the fact that a repetitive lattice structure is assumed to exist; upon elimination of the unwanted fine scale degrees of freedom which are assumed to interact linearly, one obtains the time history kernel representation $\theta(t)$. Within the MD region, the interatomic forces are nonlinear, and can be derived from any relevant potential energy function.

- We note that the time history kernel $\theta(t)$ is a matrix whose size corresponds to the minimum number of degrees of freedom in each unit cell; in three-dimensions, $\theta(t)$ is a $3 \times 3$ matrix. The compact size of $\theta(t)$ distinguishes this approach from other methods, and results from the elimination of periodically repetitive fine scale degrees of freedom. The small size of $\theta(t)$ enables us to achieve large computational savings by mathematically eliminating large portions of the lattice where an explicit atomistic representation is not desired. Specifically, even as the number of eliminated fine scale degrees of freedom grows, the size of $\boldsymbol{\theta}(t)$ remains constant.

- The final term on the right-hand side of (13), $\mathbf{R}_{0, m, n}^{\mathrm{f}}(t)$, is termed the random force, and represents the thermal forces which act on the reduced fine scale degrees of freedom due to the eliminated fine scale degrees of freedom. The random force acts to balance heat dissipated out of the MD region by the impedance force (15), and thus behaves as a heat bath term acting on the reduced MD region. The derivation and implementation of this term with regards to the bridging scale is found in the work of Karpov et al. [10], and will be discussed later in this paper.

- Due to the elimination of the fine scale degrees of freedom for the $l>0$ cells, the FE equation of motion (14) is not redundant. The coarse scale thus has two major responsibilities. The first is to capture the long wavelength nonlinear response of the system, while the impedance force described in a previous remark accounts for the high frequency waves that cannot be captured by the coarse scale. The second major role of the coarse scale relates to the coupling of the revised MD equation of motion (13) such that it depends upon the coarse scale solution $\overline{\mathbf{u}}$. It could be argued that the bridging scale is unnecessary for problems in which all of the information originates in the MD region and propagates away, because of the MD boundary conditions that were derived by Wagner et al. [16] and Karpov et al. [18]. However, because of the dependence on the coarse scale solution, information can now propagate in the reverse manner, i.e. starting in the continuum and ending up in the atomistic region. The coarse scale dependence in (13) then serves as a boundary condition on the MD simulation. The coarse scale also effects the MD simulation by controlling the motion of the ghost atoms, which exist such that the boundary atoms have a full complement of atomic neighbors. The displacements for the ghost atoms are calculated by interpolating the FE displacements of the element in which they lie. In this manner, a true two-way coupling between the coarse and fine scales is achieved. 


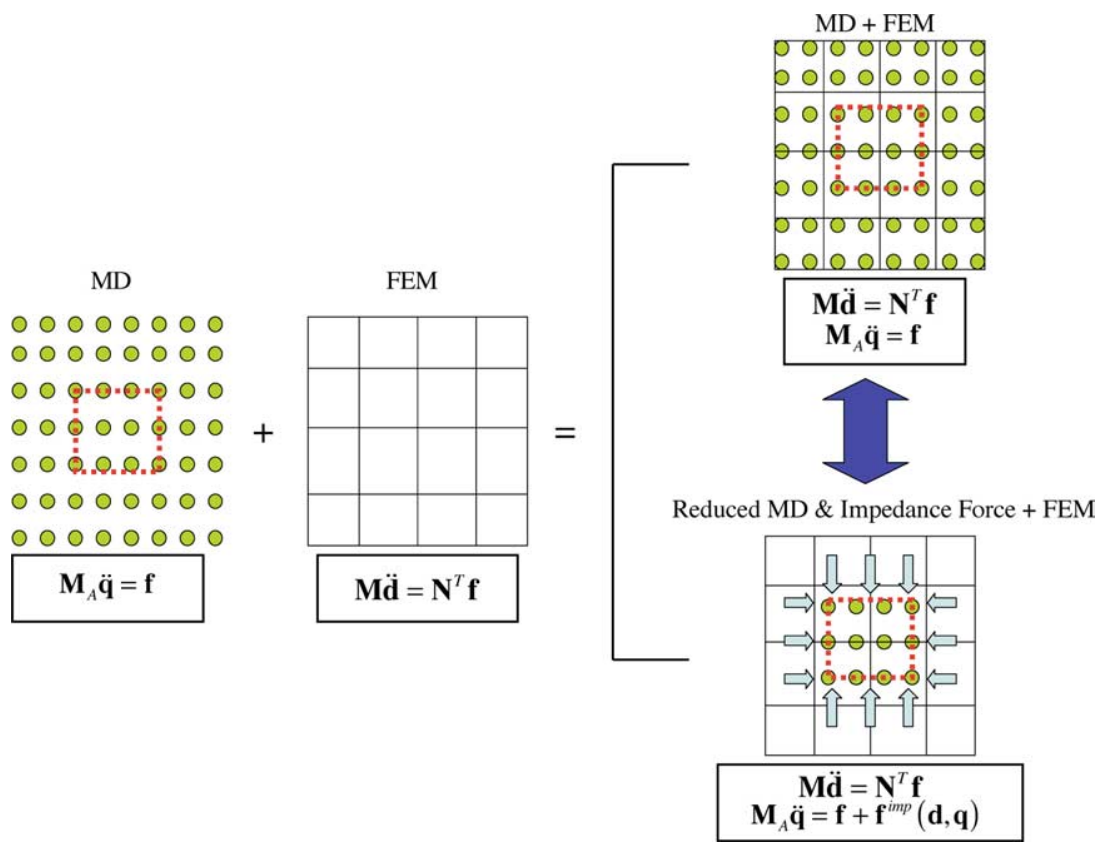

Fig. 1. Schematic illustration of bridging scale. Reprinted from [21], Copyright (2005), with permission from Elsevier.

- A schematic for the bridging scale method is shown in Fig. 1. As can be seen, the fine scale has been reduced to being active in a small portion of the domain, while the impedance force acting upon the fine scale boundaries (13) accounts for the effects of the eliminated fine scale degrees of freedom.

\section{Numerical examples}

\subsection{Finite temperature modeling of multi-scale systems}

As was discussed in the previous section, one crucial extension to the bridging scale method is in implementing the thermal forces $\mathbf{R}_{0, m, n}^{\mathrm{f}}(t)$ in (13) such that a finite temperature atomistic region can be considered. This will be possible through the recent work of Karpov et al. [10]. In that work, the random terms are modelled by utilizing the Gibbs distribution for a phonon gas along with a normal mode decomposition. The Gibbs distribution can be written as

$$
W=\frac{1}{Z} \exp \left(-\frac{H}{k_{\mathrm{B}} T}\right),
$$

where $Z$ is the thermodynamic partition function, $k_{\mathrm{B}}$ is the Boltzmann constant, and $H$ is the lattice Hamiltonian. The random component of the displacements $\mathbf{R}_{0, m, n}^{\mathrm{d}}(\tau)$ in (15) are determined by assuming a normal mode decomposition for the displacement vector. For a lattice in thermal equilibrium, it can be given as a superposition of the standing waves

$$
\mathbf{u}_{\mathbf{n}}(t)=\sum_{\mathbf{p}, s} a_{\mathbf{p}, s} \cos \left(\omega_{\mathbf{p}, s} t+\phi_{\mathbf{p}, s}\right) \mathbf{d}_{\mathbf{p}, s} \mathrm{e}^{\mathrm{ip} \cdot \mathbf{n}}
$$




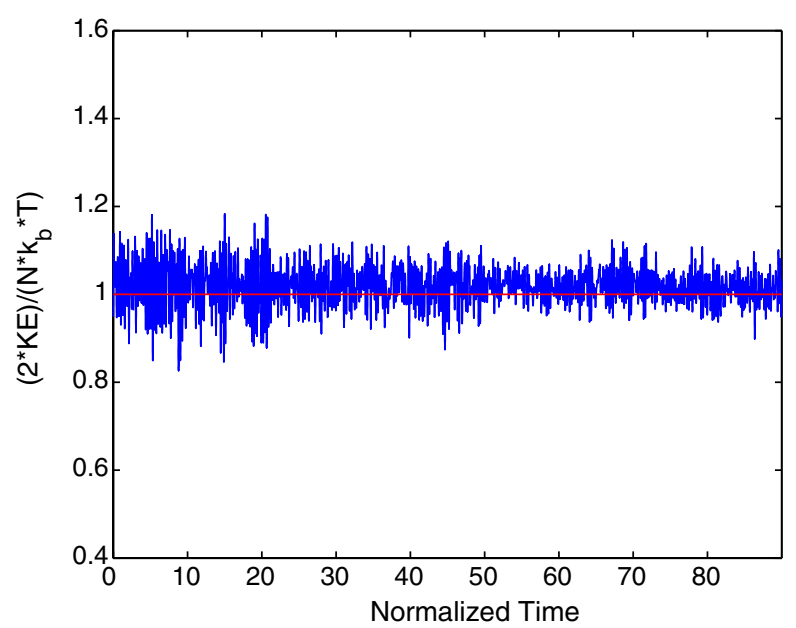

Fig. 2. Normalized kinetic energy of MD system for a target temperature of $600 \mathrm{~K}$. Values closer to one indicate a more accurate solution. Reprinted from [21], Copyright (2005), with permission from Elsevier.

where $s=1,2, \ldots, S$, and the vector $\mathbf{p}$ is comprised of the wave numbers $p_{i}=0, \pm 2 \pi / N_{i}, \ldots$, $\pm\left(N_{i}-2\right) \pi / N_{i}, \pi$. The oscillatory quantities $a_{\mathbf{p}, s} \cos \left(\omega_{\mathbf{p}, s} t+\phi_{\mathbf{p}, s}\right)$ are the normal coordinates. The values $a_{\mathbf{p}, s}$ and $\omega_{\mathbf{p}, s}$ are the normal amplitudes and normal frequencies of the lattice; $\phi_{\mathbf{p}, s}$ are initial phases. The vectors $\mathbf{d}_{\mathbf{p}, s}$ determine the direction of polarization of normal vibrations. The thermal forces $\mathbf{R}_{0, m, n}^{\mathbf{f}}(t)$ can be found by multiplying the normal displacements (17) with the lattice stiffnesses $\mathbf{K}$, as in (12).

Numerical examples demonstrate the ability of the method to perform even at high temperatures, for a single scale, atomistics-only, one-dimensional chain of atoms interacting via a nearest neighbor Morse potential. As can be seen in Fig. 2, the thermal terms $\mathbf{R}(t)$ balance the energy dissipated out of the system by the time history kernel $\theta(t)$, leaving the MD system in thermal equilibrium. The example shown is for a target system temperature of $600 \mathrm{~K}$, which is nearly half the melt temperature for this potential, demonstrating the ability of the method to perform at high temperature and thus nonlinear oscillations of the lattice. Examples utilizing this approach along with previous finite temperature bridging scale developments [19] will be shown in future work.

\subsection{Bending of carbon nanotubes}

The bridging scale method has been successfully used in modeling buckling of multi-walled carbon nanotubes. The following two sections summarize work performed by Qian et al. [14]. The slight difference in governing equations as compared to the dynamic case as well as all relevant details of the quasistatic bridging scale are given in that work, which introduces a virtual atom cluster (VAC) model for the continuum modeling of carbon nanotubes.

In these simulations, a 15-walled carbon nanotube (CNT) is considered with the outermost shell being a $(150,150)$ nanotube, and all inner shells of the $(n, n)$ type; from the outer most shell, $n$ reduces by 5 every layer. The length of the tube is $110 \mathrm{~nm}$ and the original MD system contains more than 3 million atoms. This is replaced with a system of 27,450 particles. In addition to the particles, two sections along the tube are enriched with the molecular structure of multi-walled nanotubes. The position of the enrichment region is determined by a multi-resolution analysis of the coarse scale simulation. Therefore, the scheme is adaptive. The length of each enrichment region is $3.7 \mathrm{~nm}$. Each section contains 42,000 atoms, which adds 246,000 more atomistic degrees of freedom. 


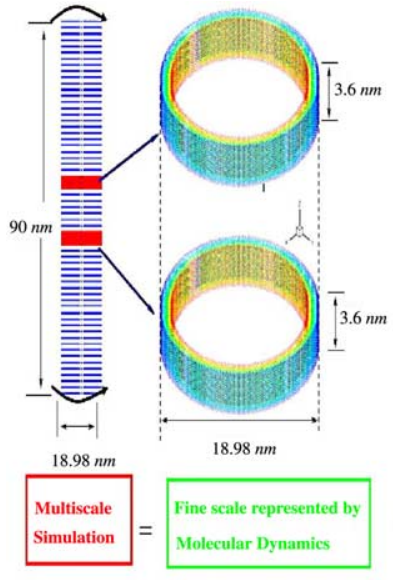

a

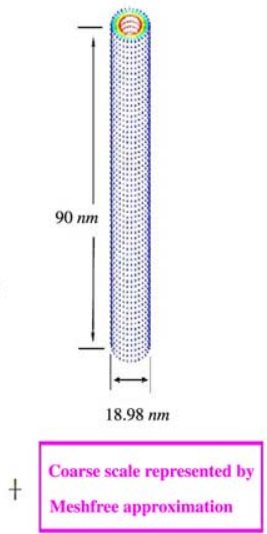

Meshfree approximation

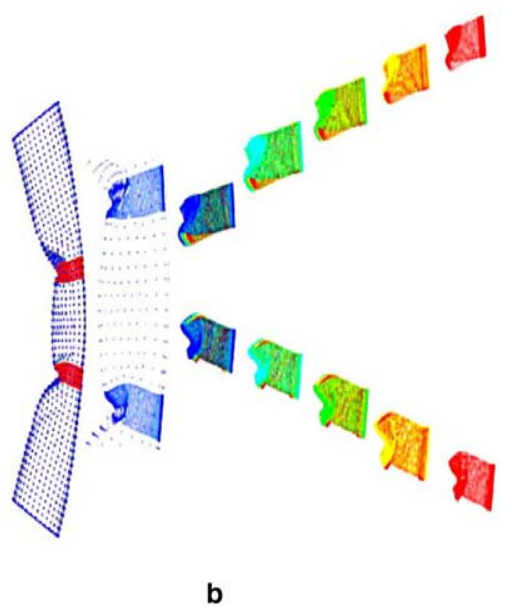

Fig. 3. Multiscale analysis of a 15 -walled CNT by the bridging scale. (a) The multi-scale simulation consists of 10 rings of carbon atoms (with 42,000 atoms each) and a meshfree continuum approximation of the 15-walled CNT by 27,450 finite element nodes. (b) The global buckling pattern is captured by the meshfree method whereas the detailed local buckling of the 10 rings of atoms are captured by the molecular dynamics simulation. Reprinted from [6], Copyright (2004), with permission from Elsevier.

A bending angle with an increment of $0.25 \%$ step is imposed on both ends of the tube for a total of 100 steps. The multi-scale configuration is illustrated in Fig. 3a. Fig. 3b shows the buckling pattern approximated by the meshfree approximation at the final stage of loading, followed by the energy density contour plot for each layer of the CNT. Two distinctive buckling patterns can be seen from the meshfree approximation, while the contour plot clearly shows the strain energy concentration at the buckling point. A unique advantage of using the multi-scale method is that the details of the molecular structure at the kinks are able to be resolved; these cannot be resolved by the coarse scale representation alone. The atomic structure of the buckling region for each layer of multi-walled CNT is plotted on the right-hand side of Fig. $3 \mathrm{~b}$.

\subsection{VAC coupling with tight binding}

Since the VAC model allows for the direct passage of information between first principles quantum mechanical approaches and FEM, a fully coupled multi-physics approach can be developed to further extend the capabilities of the present FEM/meshfree-based simulation tools. Due to the fact that the VAC model is a local representation, the resulting formulation is more computationally efficient than the fullscale first principle method and therefore the length scale limit can be further extended. The details of this extension can be found in [11].

In the following we will show a preliminary example in which the VAC model is fully coupled with a semi-empirical quantum mechanical approach called tight-binding method. The system being considered is a $(9,0)$ single-walled carbon nanotube with 360 atoms (for an overview of the mechanics of carbon nanotubes, see Qian et al. [20]). As shown in Fig. 4, the system is discretized with 60 particles using a meshfree interpolation [5]. An incremental twisting angle of $0.25^{\circ}$ step is imposed on both ends for 20 steps. For the purpose of verifying the proposed VAC model, a benchmark simulation using a full-scale tight-binding method is also performed. A comparison of the average energy of the system is shown in Fig. 5. As can be seen, the results from the VAC model closely match those from the tight-binding method. However, the VAC model is much more computationally efficient compared to a full-scale tight-binding simulation. 


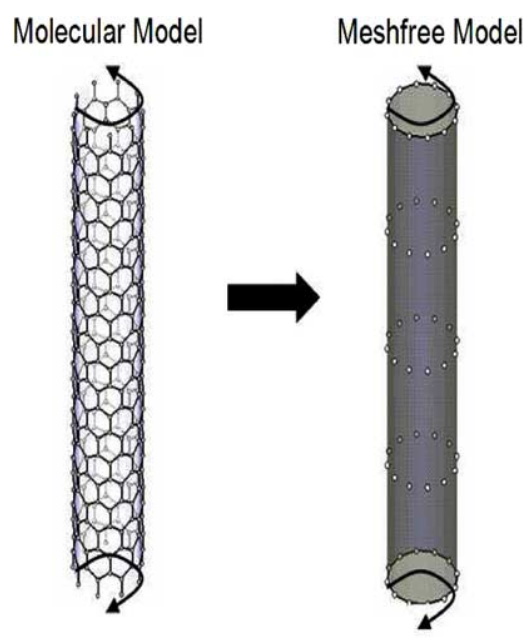

Fig. 4. Meshfree discretization of a $(9,0)$ single-walled carbon nanotube.

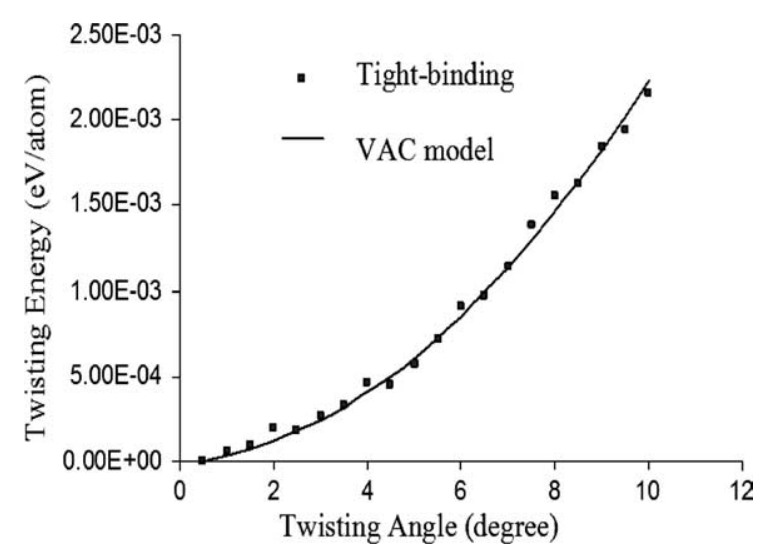

Fig. 5. Comparison of the average twisting energy between VAC model and tight-binding model.

\subsection{Two- and three-dimensional examples of dynamic fracture}

The verification of the dynamic bridging scale is done by considering dynamic fracture examples in multiple dimensions. In the first, two-dimensional benchmark problem, an atomistic pre-crack is generated in a hexagonal lattice by preventing the interaction of two neighboring rows of atoms. Thus, when the tensile waves generated by the boundary conditions applied to the continuum propagate into the atomistic region via the two-scale coupling in (13), the crack opens naturally in tension. The atoms interact via a LennardJones $6-12$ potential with nearest neighbor interactions, and model parameters $\sigma=\epsilon=1$ to simulate a model brittle solid.

A full MD simulation was also run in which the entire domain was comprised of atoms. The bridging scale simulation consisted of 91,051 atoms and 1800 finite elements, of which 900 were in the coupled MD/FE region. Correspondingly, the full MD simulation consisted of 181,201 atoms. The results for 

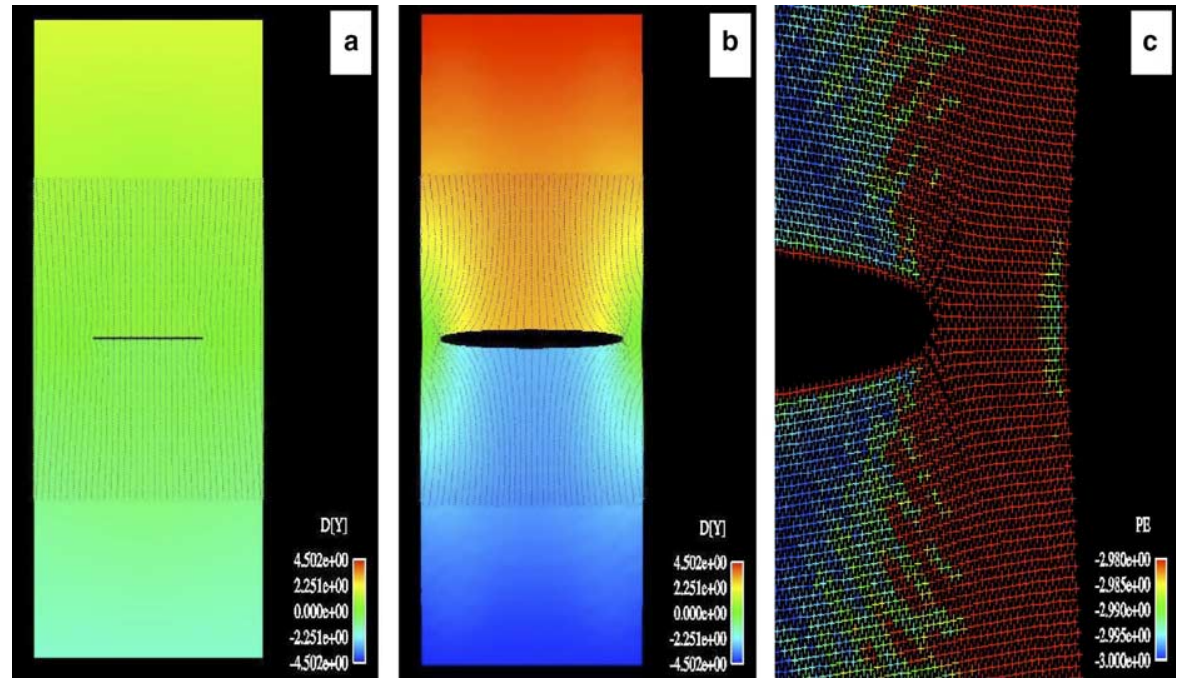

Fig. 6. (a) Initiation of atomistic crack. (b) Propagation of atomistic crack. (c) Potential energy contours ahead of atomistic crack just before complete fracture of specimen occurs. Reprinted from [6], Copyright (2004), with permission from Elsevier.

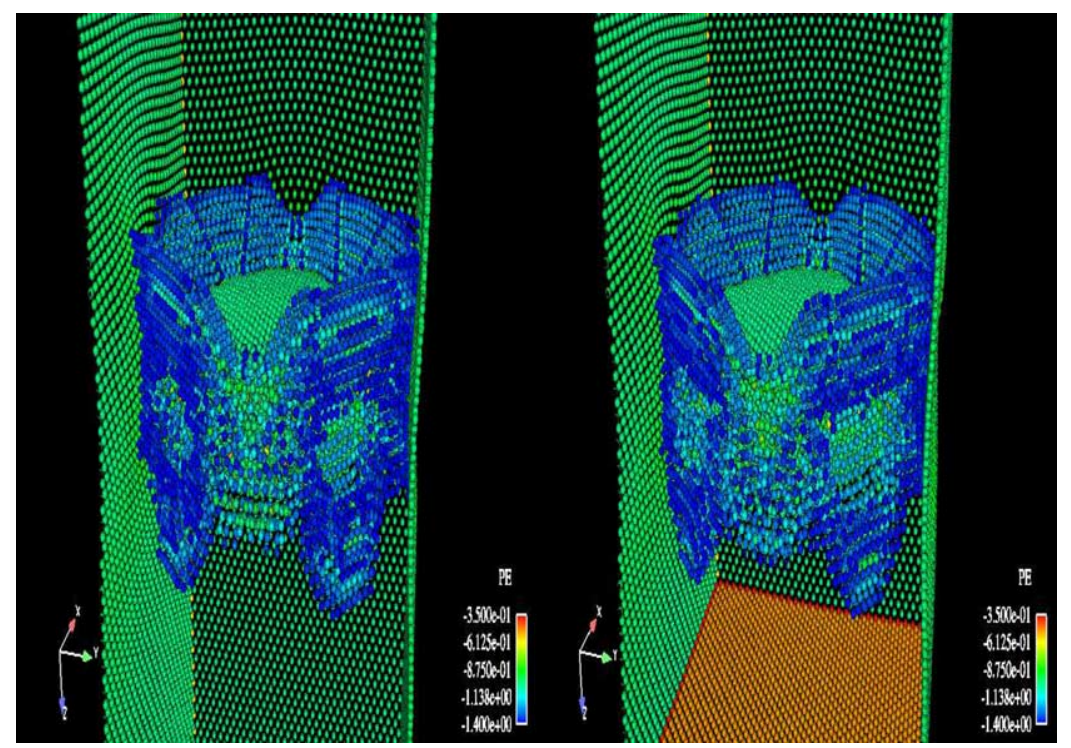

Fig. 7. Three-dimensional out of plane crack branching for (left) full MD simulation and (right) bridging scale simulation.

the bridging scale simulation are shown in Fig. 6. As can be seen, due to the two-way coupling in (13), the information originating in the continuum eventually propagates into the atomistic region, and initiates the propagation of the crack. A full atomistic simulation was performed for comparison; as shown in Park et al. [17], the bridging scale matched extremely well in terms of crack initiation time and propagation speed, all at fractions of the computational expense. 
Similar calculations have been performed in a three-dimensional FCC lattice interacting via a nearest neighbors Lennard-Jones 6-12 potential, also by Park et al. [21]. The problem description is similar to that of the two-dimensional example; the FE mesh exists everywhere, the MD region only in a small portion of the domain, and a pre-crack within the center of the MD region. As can be seen in Fig. 7, the bridging scale method again compares very well to the benchmark full MD simulation. Importantly, crucial physics of material failure such as crack branching are faithfully captured by the bridging scale simulation.

\subsection{Dynamic shear banding in a micropolar continuum}

While the bridging scale examples shown here describe the coupling of atomistic and continuum simulations, other applications of the bridging scale have successfully combined two finite element simulations or varying resolution, as in Kadowaki and Liu [22]. In that work, a fine finite element mesh is used to capture a localized region of interest, such as strain localization. Because it is extremely expensive to maintain such a fine mesh in the entire domain, the bridging scale is used to eliminate the high frequency waves emitted from the fine finite element region while coupling the coarse and fine finite element meshes.

A two-dimensional shear failure problem is solved with a micropolar continuum and the proposed bridging multi-scale method. A bar of the size of $0.4 \mathrm{~m} \times 0.04 \mathrm{~m}$ is compressed in the longitudinal direction by a prescribed velocity at both ends. The entire domain is discretized by $80 \times 8$ coarse-scale finite elements, while the central portion of $0.155 \mathrm{~m}$ is discretized by the fine-scale finite elements. The fine-scale mesh is aligned to the coarse-scale mesh near the interfaces, while it is rotated $45^{\circ}$ in the middle of the bar in order to lie in the direction of the resultant shear bands. When the mesh was not aligned to the shear band, deformation did not localize sharply.

The fine-scale mesh has 17,920 elements and $8 \times 8$ fine-scale elements occupy the same area as one coarse-scale element near the interface. All the boundaries are moment free, and the top and the bottom boundaries are stress free. Fig. 8 shows the accumulated effective plastic shear strain distribution for the regularized by the micropolar continuum. Fig. 9 shows the total shear-strain profile inside the domain of interest just after the waves created by the dynamic failure have passed away from this region. The proposed dynamic interface prevents most of the reflection of high frequency waves, while one can clearly see some reflections when the continuity of displacement is enforced at the interfaces.

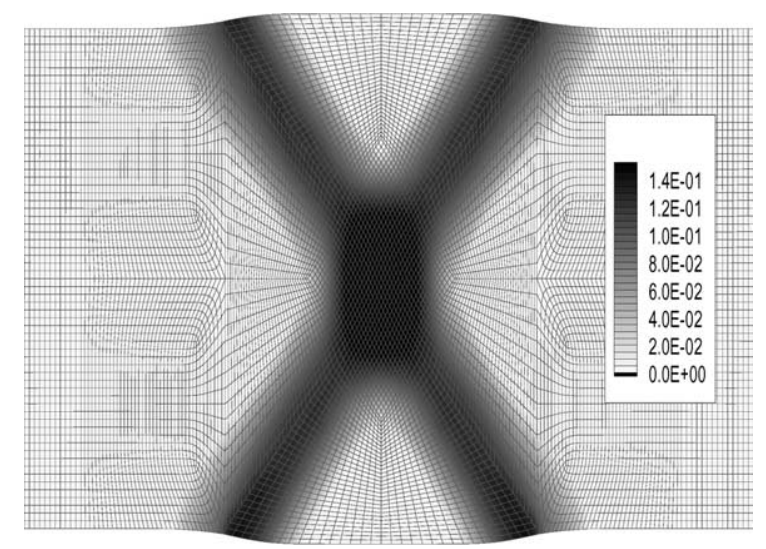

Fig. 8. Regularized solution with the micropolar continuum. Reprinted from [22], Copyright (2004), with permission from Elsevier. 

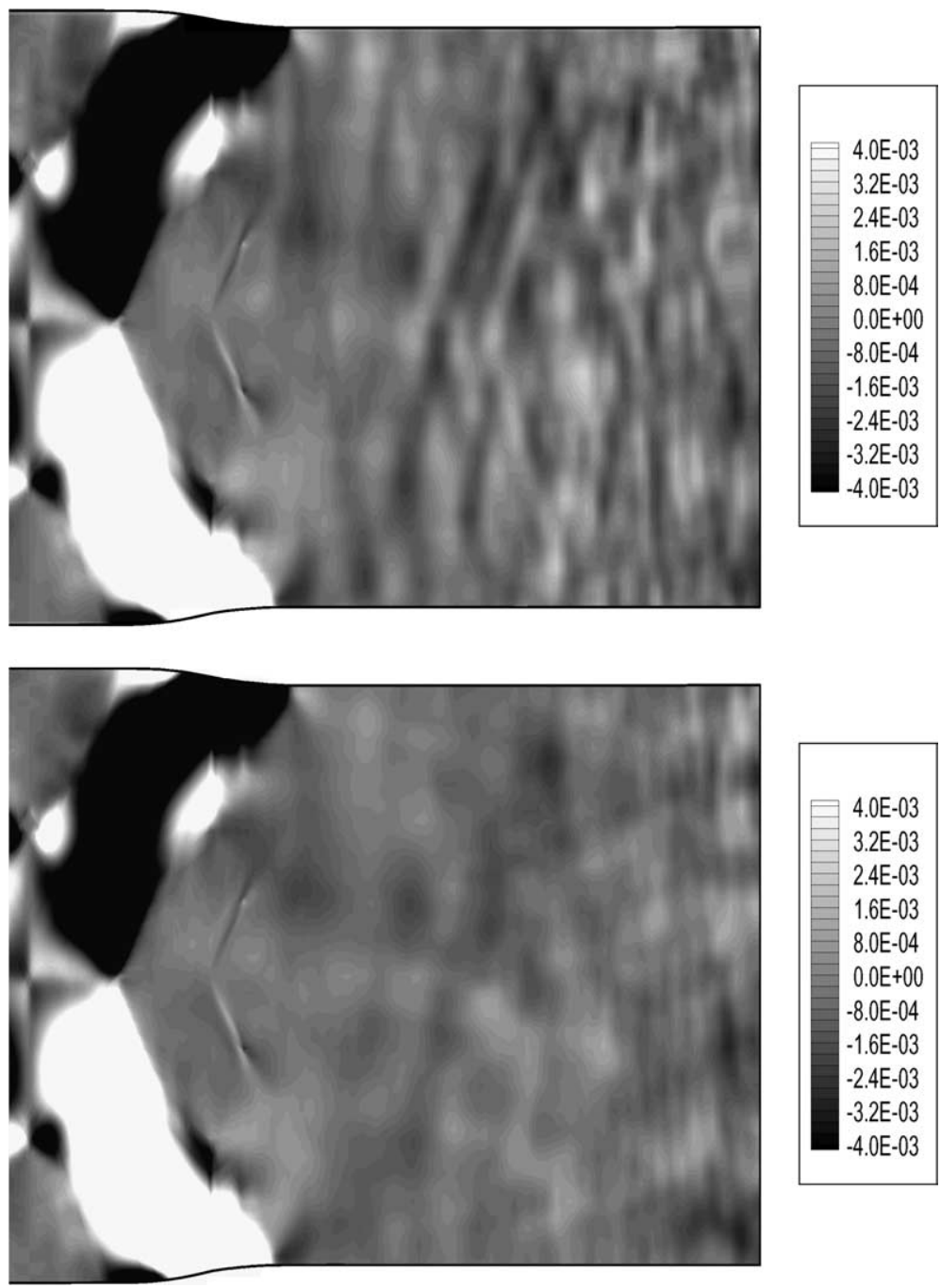

Fig. 9. Top: reflected high frequency waves when continuity of displacement is enforced at interfaces. Bottom: dramatically reduced high frequency wave reflection using correct dynamic interface condition. Reprinted from [22], Copyright (2004), with permission from Elsevier.

\section{Conclusions}

We have presented in this work a new approach termed the bridging scale for solving coupled continuum/atomistic problems. This approach offers the following distinct advantages over previously proposed methods. Firstly, there is no need to mesh the finite element region down to the atomic scale at the MD/FE border. High frequency waves which are emitted from the atomistic region are exactly accounted for in terms of a lattice impedance force which acts upon the reduced fine scale region. Finally, the method is valid not only for quasistatic analyses, but finite temperature dynamic problems.

We have validated the method on three nonlinear benchmark problems, those of quasistatic nanotube bending and buckling, dynamic fracture in hexagonal and FCC lattices, and dynamic strain localization 
in a micropolar continuum. In these problems, the fine scale is present only in a small region of the domain, in which it is crucial to capture either highly nonlinear atomistic or fine scale behavior, or to capture defects that are generated in the lattice. Despite the reduction in the size of the atomistic domains, the bridging scale simulations can be shown to faithfully reproduce the essential physics seen in a full atomistic simulation at fractions of the computational expense. The only assumption of linearity utilized in deriving the approach is contained within the time history kernel $\theta(t)$, which represents the behavior of the eliminated fine scale degrees of freedom. Inside the MD region, the interatomic forces are nonlinear, and can be obtained from any relevant potential energy function.

There are many future directions for research in this emerging area. One necessary extension is to allow non-nearest neighbor interactions in deriving the time history kernel $\theta(t)$, which represents the mathematically eliminated fine scale degrees of freedom. In this manner, realistic metallic interatomic potentials such as EAM can be incorporated into the bridging scale. Research in this direction has indeed been performed $[15,23]$, and will be incorporated into future bridging scale work.

Another crucial extension lies in the time scales that are available to the MD simulation. While we have eliminated the need to waste unnecessary time steps in updating the continuum simulation, the fact remains that the MD simulation is the bottleneck in terms of computational expense. Work is already being performed in this area by other researchers, such as in Voter et al. [24]. At some point in the future, it may be possible to combine those approaches with the bridging scale to truly extend the time scales available for the atomistic side of the simulation.

The coupling of the continuum to high accuracy atomistic, or quantum mechanical methods is work that is currently being developed by Qian and Liu [11,12]; preliminary results in this direction have been shown in this paper. This coupling of continua to quantum mechanical approaches is significant, as it extends the length scales for which the bridging scale coupling is currently valid. Research along these lines for dynamic coupling is also a future goal; in these cases, extensions of the non-reflecting MD boundary conditions developed in $[16,18,15]$ will be necessary to ensure a seamless coupling between quantum mechanics and continua.

A final, but major thrust should be to extend the method to allow finite temperature MD simulations. Because of the fact that the continuum exists everywhere, no special treatment is needed in the coupled $\mathrm{MD} / \mathrm{FE}$ region to accomodate a finite temperature MD simulation. The recently developed technique of Karpov et al. [10] can be used to regulate the temperature within the MD region. However, there is currently no established manner in which the internal energy generated within the MD region is passed or represented accurately in the continuum region. Further research is needed to develop a heat conduction equation for the coarse scale which can accurately represent the energy dissipated from the MD region due to the impedance force.

\section{Acknowledgements}

We would like to thank the National Science Foundation (NSF) and the NSF-IGERT program for their support. We would also like to thank the NSF Summer Institute on Nano Mechanics and Materials and the Army Research Office (ARO) for supporting this work.

\section{References}

[1] T.J.R. Hughes, The Finite Element Method: Linear Static and Dynamic Finite Element Analysis, Prentice-Hall, 1987.

[2] T. Belytschko, W.K. Liu, B. Moran, Nonlinear Finite Elements for Continua and Structures, John Wiley and Sons, 2002.

[3] W.K. Liu, S. Jun, Y.F. Zhang, Reproducing kernel particle methods, Int. J. Numer. Methods Fluids 20 (1995) $1081-1106$. 
[4] T. Belytschko, Y.Y. Lu, L. Gu, Element-free Galerkin methods, Int. J. Numer. Methods Engrg. 37 (1994) $229-256$.

[5] S. Li, W.K. Liu, Meshfree particle methods and their applications, Appl. Mech. Rev. 55 (2002) 1-34.

[6] W.K. Liu, E.G. Karpov, S. Zhang, H.S. Park, An introduction to computational nanomechanics and materials, Comput. Methods Appl. Mech. Engrg. 193 (2004) 1529-1578.

[7] W.A. Curtin, R.E. Miller, Atomistic/continuum coupling in computational materials science, Model. Simul. Mater. Sci. Engrg. 11 (2003) R33-R68.

[8] T. Hughes, G. Feijoo, L. Mazzei, J. Quincy, The variational multiscale method-a paradigm for computational mechanics, Comput. Methods Appl. Mech. Engrg. 166 (1998) 3-24.

[9] G.J. Wagner, W.K. Liu, Coupling of atomistic and continuum simulations using a bridging scale decomposition, J. Comput. Phys. 190 (2003) 249-274.

[10] E.G. Karpov, H.S. Park, W.K. Liu, Phonon heat bath for crystalline solids, preprint;

W.K. Liu, E.G. Karpov, H.S. Park, Nano Mechanics and Materials: Theory, Multiscale Methods and Applications, John Wiley \& Sons, 2005, in press.

[11] D. Qian, W.K. Liu, A cluster model for integrating quantum-mechanical descriptions at multiple length scales, in preparation.

[12] D. Qian, R.H. Rondhalekar, E.G. Karpov, W.K. Liu, Multiscale analysis of nonlinear mechanics in nanoscale materials, in: Z.H. Yao, M.W. Yuan, W.X. Zhong (Eds.), Proceedings of the 6th World Congress of Computational Mechanics, Tsinghua University Press and Springer-Verlag, 2004, p. 185.

[13] H.S. Park, W.K. Liu, Introduction and tutorial on multiple scale analysis in solids, Comput. Methods Appl. Mech. Engrg. 193 (2004) 1733-1772.

[14] D. Qian, G.J. Wagner, W.K. Liu, A multiscale projection method for the analysis of carbon nanotubes, Comput. Methods Appl. Mech. Engrg. 193 (2004) 1603-1632.

[15] H.S. Park, E.G. Karpov, W.K. Liu, Non-reflecting boundary conditions for atomistic, continuum and coupled atomistic/ continuum simulations, Int. J. Numer. Methods Engrg. 64 (2005) 237-259.

[16] G.J. Wagner, E.G. Karpov, W.K. Liu, Molecular dynamics boundary conditions for regular crystal lattices, Comput. Methods Appl. Mech. Engrg. 193 (2004) 1579-1601.

[17] H.S. Park, E.G. Karpov, W.K. Liu, P.A. Klein, The bridging scale for two-dimensional atomistic/continuum coupling, Philos. Mag. 85 (1) (2005) 79-113.

[18] E.G. Karpov, G.J. Wagner, W.K. Liu, A Green's function approach to deriving non-reflecting boundary conditions in molecular dynamics simulations, Int. J. Numer. Methods Engrg. 62 (2005) 1250-1262.

[19] H.S. Park, E.G. Karpov, W.K. Liu, A temperature equation for coupled atomistic/continuum simulations, Comput. Methods Appl. Mech. Engrg. 193 (2004) 1713-1732.

[20] D. Qian, G.J. Wagner, W.K. Liu, M.F. Yu, R.S. Ruoff, Mechanics of carbon nanotubes, Appl. Mech. Rev. 55 (6) (2002) $495-533$.

[21] H.S. Park, E.G. Karpov, P.A. Klein, W.K. Liu, Three-dimensional bridging scale analysis of dynamic fracture, J. Comput. Phys. 207 (2005) 588-609.

[22] H. Kadowaki, W.K. Liu, Bridging multi-scale method for localization problems, Comput. Methods Appl. Mech. Engrg. 193 (2004) 3267-3302.

[23] P.A. Klein, J.A. Zimmerman, Coupled atomistic-continuum simulation using arbitrary overlapping domains, J. Comput. Phys., in press.

[24] A.F. Voter, F. Montalenti, T.C. Germann, Extending the time scale in atomistic simulation of materials, Annu. Rev. Mater. Res. 32 (2002) 321-346. 